\title{
Direct Evidence of Symmetry between Bilateral Human Corneas in Biomechanical Properties: A Comparison Study with Fresh Corneal Tissue
}

\author{
Chao Xue $\mathbb{D}^{1,2}$ Yaoqi Xiang, ${ }^{3}$ Yi Song, ${ }^{1,2}$ Min Shen, ${ }^{3}$ Di Wu, ${ }^{1,2}$ and Yan Wang $\mathbb{D}^{1,2}$ \\ ${ }^{1}$ Clinical College of Ophthalmology, Tianjin Medical University, Tianjin, China \\ ${ }^{2}$ Tianjin Eye Hospital, Tianjin Key Laboratory of Ophthalmology and Visual Science, Tianjin Eye Institute, Tianjin, China \\ ${ }^{3}$ School of Mechanical Engineering, Tianjin University, Tianjin, China
}

Correspondence should be addressed to Yan Wang; wangyan7143@vip.sina.com

Received 27 August 2020; Revised 18 December 2020; Accepted 13 January 2021; Published 20 January 2021

Academic Editor: Paolo Fogagnolo

Copyright (c) 2021 Chao Xue et al. This is an open access article distributed under the Creative Commons Attribution License, which permits unrestricted use, distribution, and reproduction in any medium, provided the original work is properly cited.

Purpose. To investigate the difference between the eyes from the same human with respect to the biomechanical properties of fresh corneal tissues and investigate the assumption of similarity of the corneal biomechanical properties between the eyes. Methods. Strip specimens extracted through a small incision lenticule extraction (SMILE) surgery were tested using a uniaxial tensile test. The specimens were extracted vertically. Low-strain tangent modulus (LSTM), high-strain tangent modulus (HSTM), and tensile strength $\left(\sigma_{b}\right)$ were the biomechanical parameters used in the comparison of the eyes from the same human. Results. Ninety corneal specimens from 45 persons were included in this study. The LSTM of the left and right eyes were $1.34 \pm 0.52$ and $1.37 \pm 0.46 \mathrm{MPa}$, while the HSTM were $50.53 \pm 7.51$ and $49.41 \pm 7.01 \mathrm{MPa}$, respectively. There was no significant difference between the eyes in terms of LSTM, HSTM, and $\sigma_{b}(P=0.813,0.335$, and 0.605 , resp.). The LSTM and HSTM were significantly correlated with the spherical equivalent (SE) $(P \leq 0.01, P=0.001$, resp.). Conclusions. The assumption that the corneal biomechanical properties of the eyes from the same human are similar has been confirmed for the first time using fresh human corneal tissue. This finding may be useful in further biomechanical studies.

\section{Introduction}

A transparent cornea is an important component of the outer ocular tunic. It accounts for approximately two-thirds of the optical power of the eye and serves as an effective protective shield. Disease, trauma, surgery, and contact lenses can affect its focusing ability due to the changes in its shape. Therefore, it is very important to understand how to predict the response of the cornea to external changes. Measurement of the corneal biomechanical properties can contribute to early diagnosis of corneal dilatation, keratoconus, and other corneal diseases, and it can be used to evaluate the efficacy of certain clinical treatments, such as corneal refractive surgery and corneal cross-linking therapy [1-4]. Owing to the above reasons, investigations of the corneal biomechanical properties have become even more important in recent years.
Previous studies of corneal biomechanical properties were conducted using animals' and cadavers' eyes or pathological cornea because of the preciousness of the human cornea. [5, 6] However, these studies could not accurately describe the material properties of the normal human corneal tissue. With the development of small incision lenticule extraction (SMILE) surgery, it is now possible to obtain fresh human corneal tissue; this has made the direct study of the fresh human cornea a reality.

The mechanical behavior of cornea is determined by the stroma, which comprises about $90 \%$ of the cornea's thickness. The stroma is made up of several hundred lamellae, each composed of parallel collagen fibrils embedded within an extracellular matrix $[7,8]$. Earlier microstructural studies have found that the majority of collagen fibrils in the central region of human corneas had a preferential orientation in 
the inferior-superior (vertical) or nasal-temporal (horizontal) directions [9]. Because collagen fibrils are the main load-carrying elements of the stroma, their preferential orientation may lead to anisotropic mechanical behavior of the cornea $[5,10,11]$.

A uniaxial tensile test is a classic method in the study of anisotropy. The behavior patterns of corneal tissue strips extracted from different anatomical directions have been compared in previous research $[5,12]$. The basis of this methodology is the assumption of symmetry (about the body midline) in the microstructure and topography of the two corneas that were found in earlier studies [13, 14]. However, these studies do not provide direct evidence of a similarity in the corneal biomechanical behavior of the eyes from the same human. Elsheikh and Alhasso [12] found a proof of symmetry of the corneal biomechanical behavior in the eyes in a previous study; however, the study was only a qualitative comparison in porcine eyes. Further information from quantitative tests in human corneas is imperative.

The purpose of this study was to investigate the assumption of similarity of the corneal properties of eyes from the same human so as to guide future studies. It was conducted as a part of the efforts to explore the biomechanical behavior of human cornea.

\section{Materials and Methods}

2.1. Surgical Procedures and Specimen Preparation. All operations were performed by the same experienced surgeon. The VisuMax femtosecond laser system (Carl Zeiss Meditec AG, Jena, Germany) was used for the operations. Details of the surgical procedure have been described in previous studies [15]. The thickness of the cap was $120 \mu \mathrm{m}$. After the creation of the refractive lenticule and small incision, the surgeon dissected the lenticule from the surrounding tissue and extracted it through the small incision [16].

After the cornea lenticule was extracted through the SMILE surgery, the 12 o'clock position was marked with gentian violet for orientation of the superior-inferior cut, and then the lenticule was preserved in storage medium, Eusol-C (Alchimia, Padova, Italy); the storage was in a refrigerator kept at a temperature below $4^{\circ} \mathrm{C}$ for no more than 24 hours, during which time the lenticules were taken out and prepared for the experiment one by one. The lenticule was gently placed on a rubber base and was cut to a $1.0 \mathrm{~mm}$ wide strip specimen from the central region with a custom-made double-blade knife in a superior-inferior (vertical) direction.

All patients underwent a systematic ophthalmologic examination, including slit-lamp microscopy, corneal topography, and intraocular pressure (IOP) measurement, to ensure a healthy cornea. Exclusion criteria were keratoconus or suspected keratoconus, active ocular and systemic disease, prior clinical history of ocular surgery or trauma, and any other condition that can affect the health of the cornea [16].

The study was approved by the Ethics Committee of Tianjin Eye Hospital (201922), Tianjin Medical University, and adhered to the tenets of the Declaration of Helsinki.
Informed consent was obtained from each patient to use any clinical data for analysis and publication.

2.2. Uniaxial Tensile Testing. The specimens were then clamped between the jaws with rough thread surfaces to avoid slippage. Next, they were subjected to uniaxial tensile tests starting with 3 loading/unloading cycles and followed by loading to failure. We found that the hysteresis loop decreased with successive cycles and eventually disappeared (Figure 1). After 3 cycles, the load-displacement curve became stable, and the specimens were regarded as preconditioned. The tests were performed using an In Situ Bidirectional Tension and Compression Testing System (IBTC-50, Tianjin Care Measure and Control Co., Ltd., Tianjin, China) in a water bath apparatus filled with normal saline at room temperature. The strain rate was $0.01 \mathrm{~mm} / \mathrm{s}$ [16].

Figure 2 is a typical stress-strain curve that can be divided into four sections. In the first part of the OA segment, very small changes in load are associated with rapid increases in the deformation; the OA segment is a low-strain region, and it can be regarded as a linear elastic region. In the $\mathrm{AB}$ segment, the load increases exponentially with an increase in deformation, and this produces a nonlinear relationship. The BC segment is a high-strain region and approximately a straight line. $D$ is the fracture point [16].

2.3. The Mathematical Analysis Procedure. At the beginning of the experiment, the clamped specimen in the curled state was not fully stretched. After three preconditioned loading/ unloading cycles, the cornea was completely stretched while the load served to zero. Displacement in this period of time was recorded as $d_{0}$. Therefore, the length of the specimen at the beginning of the uniaxial tensile test was calculated as follows: $l_{0}=d_{0}+1.5$.

$1.5 \mathrm{~mm}$ was the distance between the two clamps.

The elongation recorded by the machine was converted to strain using the following formula:

$$
\varepsilon=\frac{d-d_{0}}{l_{0}},
$$

where $d$ is the elongation of cornea specimen.

Stress was calculated as follows:

$$
\sigma=\frac{F}{\omega \cdot L T}
$$

where F is load; LT is the central thickness of cornea specimen, which was obtained from the SMILE surgery data; and wis the specimen width, which was $1 \mathrm{~mm}$ in this experiment.

Elastic modulus $(E)$ is the tangent slope of the stressstrain curve; the following equation was used to derive it [12]:

$$
E=\frac{\Delta \sigma}{\Delta \varepsilon}
$$




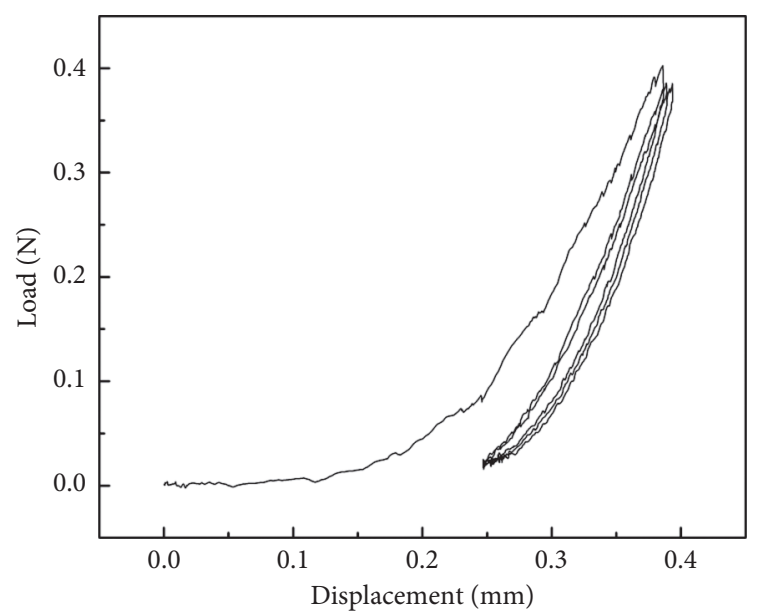

FIgURE 1: Load-displacement curve during the preconditioning process.

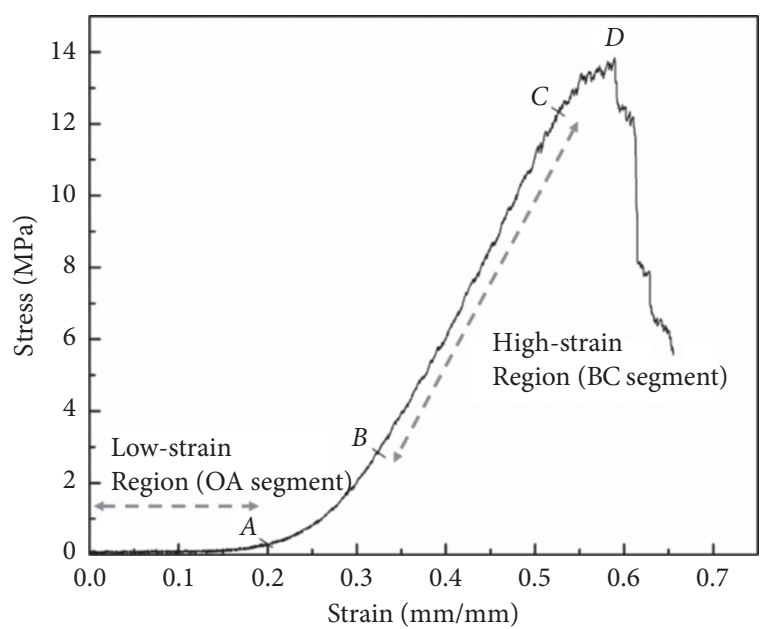

Figure 2: The stress-strain curve of a corneal strip [16].

The elastic moduli of the $\mathrm{OA}$ and $\mathrm{BC}$ segments were defined as low-strain tangent modulus (LSTM) and highstrain tangent modulus (HSTM), respectively [17].

Tensile strength was calculated as follows [18]:

$$
\sigma_{b=} \frac{F_{b}}{\omega \cdot L T}
$$

where $F_{b}$ is the maximum load at the fracture point.

LSTM, HSTM, and tensile strength $\left(\sigma_{b}\right)$ were used as parameters for the comparison of the left and right eye.

2.4. Statistical Analysis. Statistical analysis was performed using IBM SPSS Statistics Software (version 20.0, IBM Corp., Armonk, NY). Comparison between the two eyes was performed using the paired $t$-test or nonparametric test. In addition, the Pearson or Spearman correlation test was used to assess correlations between the corneal biomechanical parameters and related factors. A $P$ value of less than 0.05 was considered statistically significant.

\section{Results}

Ninety corneal lenticules from 45 persons were included in this study. There were 24 male and 21 female patients, and the mean age was $21.02 \pm 3.74$ (range, 16-31) years. Demographic data are summarized in Table 1; all data were normally distributed. There was no significant difference between the eyes with respect to any of the parameters.

Figure 3 shows a comparison of fitting curves for left and right eyes. They had similar stress-strain curves under the same stress or strain; only slight differences between the eyes from the same human were observed. Comparison of the eyes with respect to low-strain tangent modulus (LSTM), high-strain tangent modulus (HSTM), and tensile strength $\left(\sigma_{b}\right)$ is shown in Table 2; there was no significant difference between the eyes in terms of all three parameters.

The frequency distribution of LSTM and LSTM in the 90 eyes is shown in Figure 4, which shows that HSTM had general normal distributions while LSTM showed a skewed distribution. The correlations among LSTM, HSTM, and related parameters were further analyzed. The results showed that LSTM and HSTM were significantly correlated with SE; the $P$ values were $\leq 0.01$ and 0.001 , respectively (Table 3).

\section{Discussion}

A lot of research has been done in recent years to study the importance of corneal biomechanics, and significant progress has been achieved. However, due to the preciousness of human cornea, it was impossible to carry out experimental studies on fresh corneal materials. A large number of clinical studies have proved that SMILE surgery produces safe, effective, predictable, and stable outcomes in the correction of myopia and astigmatism [19-21]. In addition, for the study of corneal biomechanics, this operation provides fresh corneal material; and this has created a new stage in corneal biomechanics research.

A comparison of eyes from the same human is based on the assumption of microstructural and topographic symmetry [13, 14, 22-24]. Elsheikh et al. [12] found qualitative evidence of similarity in the corneal behavior of porcine eyes. However, there is no direct evidence of the similarity in biomechanical properties of the cornea in human eyes. The purpose of the present study was to find this evidence.

Eusol-C was used to preserve the corneal lenticule immediately after it was surgically extracted. The effectiveness of this storage medium in maintaining stromal hydration after 7 days of storage has been confirmed in previous studies $[5,25]$. In the present study, the corneal lenticule was preserved in the storage medium, Eusol-C, for no more than 24 hours so that the specimens would maintain their good quality for the tests. Although the tensile tests were conducted in saline solution in the present study, the experiment was accomplished in a very short time; therefore, it is unlikely that significant swelling occurred during the experiments. [16].

The classical uniaxial tensile test was used to compare the eyes with respect to the low-strain tangent modulus (LSTM), 
TABLE 1: Demographics data of the specimens.

\begin{tabular}{lccc}
\hline Parameters & Left eye & Right eye & $P$ value \\
\hline CCT $(\mu \mathrm{m})$ & $551.44 \pm 23.99$ & $552.00 \pm 22.43$ & 0.574 \\
SE $(\mathrm{D})$ & $-5.48 \pm 1.44$ & $-5.57 \pm 1.72$ & 0.437 \\
IOP $(\mathrm{mm} \mathrm{Hg})$ & $16.38 \pm 1.91$ & $16.42 \pm 1.94$ & 0.862 \\
LT $(\mu \mathrm{m})$ & $111.09 \pm 23.11$ & $112.27 \pm 26.33$ & 0.512 \\
\hline
\end{tabular}

$\mathrm{CCT}=$ central corneal thickness; $\mathrm{SE}=$ spherical equivalent; $\mathrm{IOP}=$ intraocular pressure; $\mathrm{LT}=$ central thickness of cornea specimen.

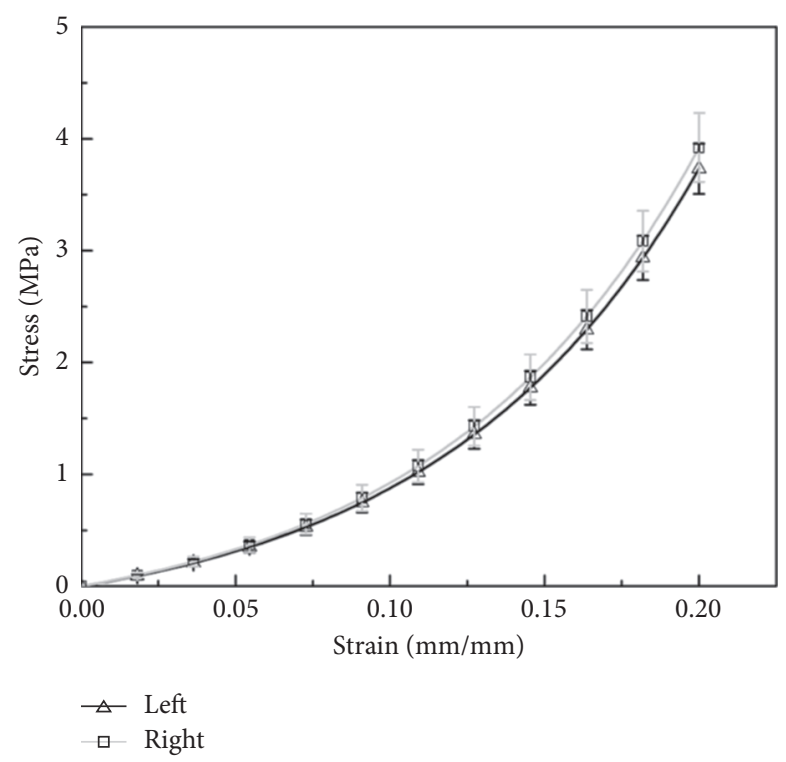

FIGURE 3: Stress-strain measurements of left and right eyes.

TABLE 2: Comparison of left and right eyes.

\begin{tabular}{lccc}
\hline Parameters & Left eye & Right eye & $P$ value \\
\hline${\text { LSTM }(\mathrm{MPa})^{*}}_{\text {HSTM }(\mathrm{MPa})}$ & $1.34 \pm 0.52$ & $1.37 \pm 0.46$ & 0.813 \\
$\sigma_{b}(\mathrm{MPa})$ & $50.53 \pm 7.51$ & $49.41 \pm 7.01$ & 0.335 \\
\hline
\end{tabular}

${ }^{*}$ Nonparametric test.

high-strain tangent modulus (HSTM), and tensile strength $\left(\sigma_{b}\right)$. Eyes from the same human had similar stress-strain curves, and statistical analysis showed that there was no statistically significant difference between the eyes. These quantitative data demonstrated directly that there is no obvious difference between the two human corneas with respect to in the biomechanical properties. In other words, the two human corneas are symmetrical. Results of this study are the first direct evidence of binocular biomechanical symmetry.

Elastic modulus is an important parameter in the measurement of biomechanical properties, and different values have been reported in previous studies [18, 26-28]. The range of values is surprisingly large in these studies, from $0.159 \mathrm{MPa}$ [27] to $57 \mathrm{MPa}$ [28]. Glass et al. [29] have given an explanation for this phenomenon. Compared to these previous studies, the LSTM of the left and right eye were $1.34 \pm 0.52$ and $1.37 \pm 0.46 \mathrm{MPa}$, while the HSTM were
$50.53 \pm 7.51$ and $49.41 \pm 7.01 \mathrm{MPa}$, respectively, in the present study; these values were relatively high compared with the values from previous studies. There are several possible reasons for this finding. Firstly, cadavers' eyes or pathological cornea were used in the previous studies, and these specimens may have had changes in the biomechanical properties of the cornea due to tissue degradation. The specimens used in this study were from the fresh normal human corneal tissue, and this may explain the difference. Secondly, previous studies $[30,31]$ have shown that the epithelium and Bowman's layer do not contribute significantly to the mechanical stability of the cornea although they comprise most of the corneal thickness outside the stroma. Labate et al. [32] have shown that the elastic modulus within the stroma decreases from the anterior to posterior part; in other words, the anterior stroma has the highest elastic modulus in the cornea. Therefore, for specimens of the same thickness, the biomechanical properties of a specimen 


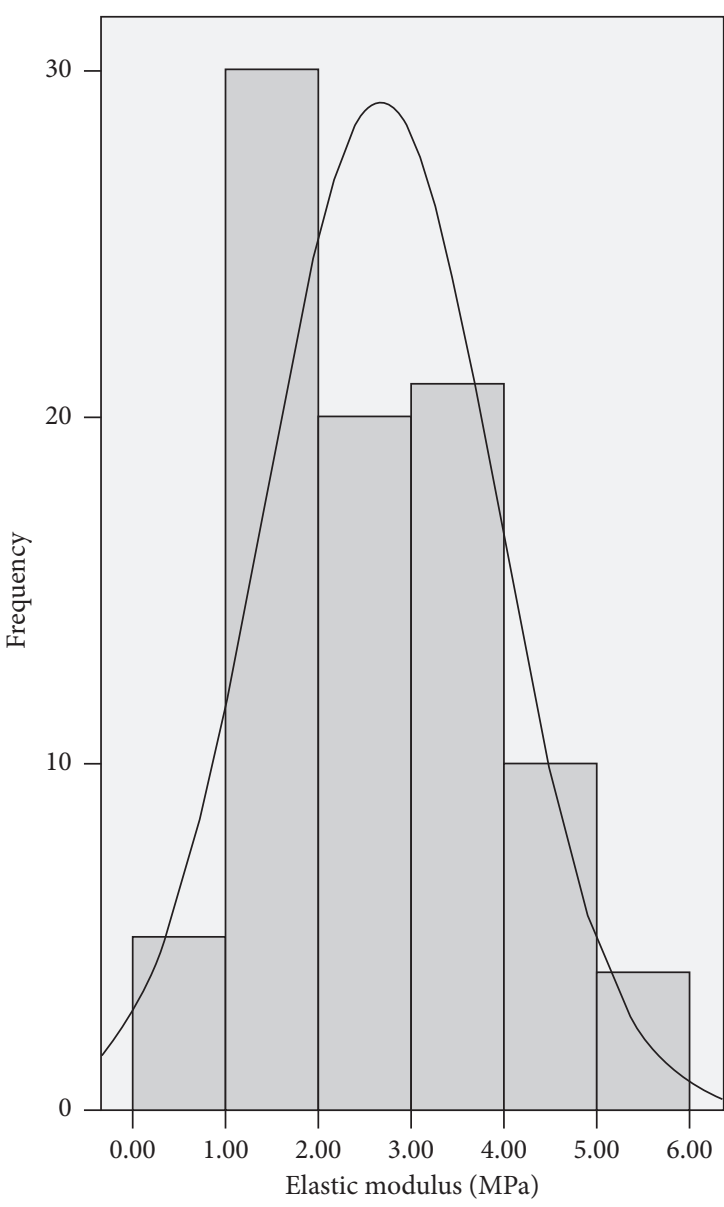

(a)

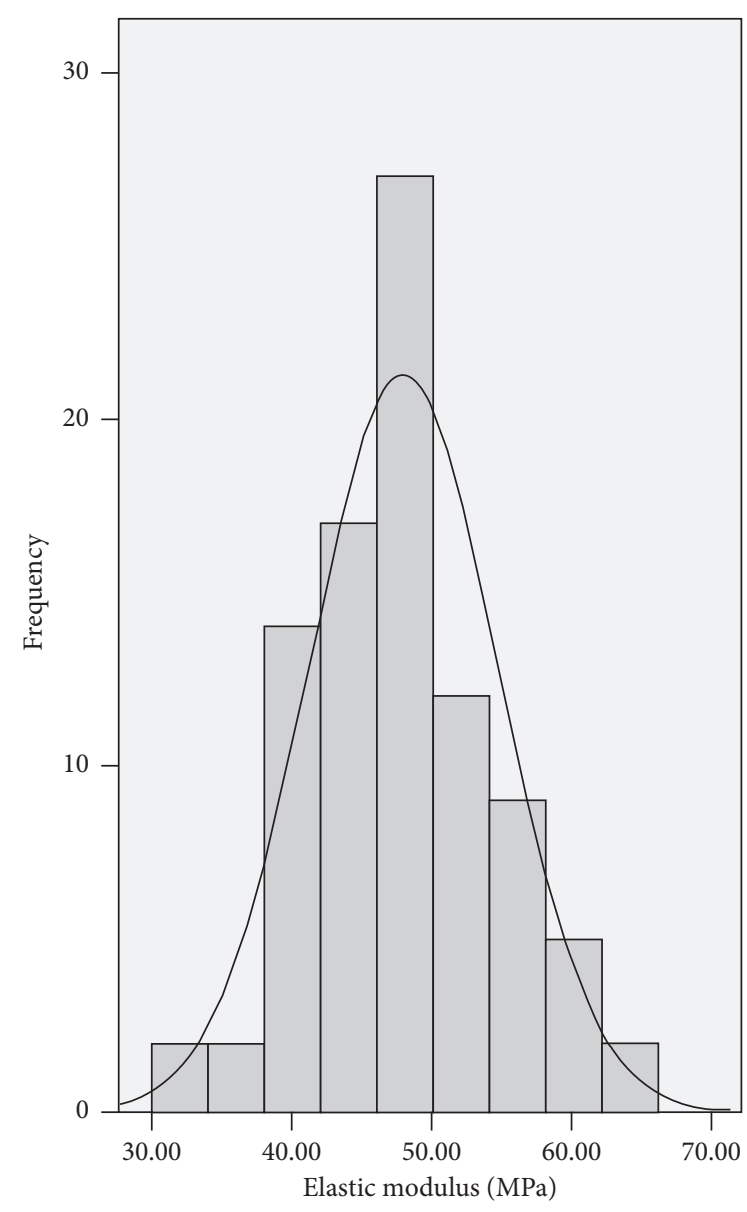

(b)

Figure 4: Frequency distribution histogram of LSTM (a) and HSTM (b).

TABLE 3: Correlation coefficient of corneal biomechanical parameters and relevant influencing parameters.

\begin{tabular}{lcccccc}
\hline Parameters & Gender & Age & CCT & SE & IOP & LT \\
\hline LSTM & 0.062 & -0.013 & 0.002 & $0.367^{*}$ & 0.007 & $-0.397^{*}$ \\
HSTM & 0.129 & 0.040 & 0.083 & $-0.358^{*}$ & 0.095 & $0.379^{*}$ \\
\hline
\end{tabular}

${ }^{*} P<0.05$.

comprised of the anterior stroma are better than those of a specimen comprising the whole corneal thickness. Because the thickness of the cap was $120 \mu \mathrm{m}$ in the SMILE surgery in this study, the specimen was extracted only from the anterior stroma of the cornea; this specimen may have better biomechanical properties than the full-thickness cornea, and it may have a higher elastic modulus than the full-thickness cornea used in the previous studies. In addition, we found that LSTM was much smaller than LSTM. As a soft tissue, cornea has viscoelastic properties; therefore, the tangent modulus increases with an increase in stress [12].

The LSTM and HSTM distributions of the 90 corneas were plotted, and it was observed that HSTM had general normal distributions while LSTM showed a skewed distribution. The relationship between biomechanical characteristics and relevant influencing parameters were analyzed.
The results showed that LSTM and HSTM were significantly correlated with SE. Matalia et al. [33] found that corneal stiffness (CS) correlated negatively with the grade of myopia. Additional work showed that subjects with high myopia had lower normalized corneal tangent moduli than subjects with low myopia had [34-36]. The correlation of LSTM and HSTM with SE in the present experiment was consistent with these studies. The LSTM represents biomechanical behavior within the physiological state, whereas the HSTM occurs beyond the physiological state; this difference may be the reason why LSTM was positively correlated with SE whereas HSTM was negatively correlated with SE, and it needs a further study.

Elsheikh et al. [27] found that age was positively correlated with corneal biomechanics in their study. This was not observed in the present study; this may be because the 
sample in this study was comprised mainly of young people aged 16 to 31 years. The age difference was small in the study population; this may explain the insignificant correlation of age with the biomechanical characteristics that were observed in this study.

There are a few limitations in the present study. The sample size was relatively small, and the population comprised mainly young people. The small size of the lenticule makes it difficult to measure the thickness of the specimen, and we could only use the theoretical central lenticule thickness to calculate stress. However, the results remain viable because the focus was on the comparison between the two corneas of the same human.

In conclusion, to the best of our knowledge, the present study is the first to provide quantitative proof of symmetry of the biomechanics properties of human eyes by using fresh human corneal tissue. These findings could be useful for further studies. Further studies with larger sample sizes are needed to understand the biomechanical properties of human cornea.

\section{Data Availability}

The data that support the findings of this study are available from the corresponding author upon reasonable request (wangyan7143@vip.sina.com).

\section{Disclosure}

Chao Xue, Yaoqi Xiang, and Yi Song are co-first authors.

\section{Conflicts of Interest}

The authors report no conflicts of interest.

\section{Acknowledgments}

This work was supported by the National Natural Science Foundation of China (Grants no. 81670884). The authors thank Professor Haixia Zhang and Lin Li Professor from School of Biomedical Engineering, Beijing Key Laboratory of Fundamental Research on Biomechanics in Clinical Application, Capital Medical University, for giving advice and help in the experiment and data processing method in this study.

\section{References}

[1] S. Kling and F. Hafezi, "Corneal biomechanics - a review," Ophthalmic and Physiological Optics, vol. 37, no. 3, pp. 240252, 2017.

[2] D. P. Piñero and N. Alcón, "Corneal biomechanics: a review," Clinical and Experimental Optometry, vol. 98, no. 2, pp. 107-116, 2015.

[3] J. W. Ruberti, A. Sinha Roy, and C. J. Roberts, "Corneal biomechanics and biomaterials," Annual Review of Biomedical Engineering, vol. 13, no. 1, pp. 269-295, 2011.

[4] F. Cavas, D. Piñero, J. S. Velázquez et al., "Relationship between corneal morphogeometrical properties and biomechanical parameters derived from dynamic bidirectional air applanation measurement procedure in keratoconus," Diagnostics (Basel), vol. 10, no. 9, 2020.

[5] A. Elsheikh, M. Brown, D. Alhasso, P Rama, M Campanelli, and D Garway-Heath, "Experimental assessment of corneal anisotropy," Journal of Refractive Surgery (Thorofare, N.J. :1995), vol. 24, no. 2, pp. 178-187, 2008.

[6] A. Elsheikh, D. Alhasso, and P. Rama, "Biomechanical properties of human and porcine corneas," Experimental Eye Research, vol. 86, no. 5, pp. 783-790, 2008.

[7] L. J. Müller, E. Pels, L. R. H. M. Schurmans, and G. F. J. M. Vrensen, "A new three-dimensional model of the organization of proteoglycans and collagen fibrils in the human corneal stroma," Experimental Eye Research, vol. 78, no. 3, pp. 493-501, 2004.

[8] D. A. D. Parry and A. S. Craig, "Electron microscope evidence for an $80 \AA$ unit in collagen fibrils," Nature, vol. 282, no. 5735, pp. 213-215, 1979.

[9] C. R. Ethier, M. Johnson, and J. Ruberti, "Ocular biomechanics and biotransport," Annual Review of Biomedical Engineering, vol. 6, no. 1, pp. 249-273, 2004.

[10] M. Singh, J. Li, Z. Han et al., "Assessing the effects of riboflavin/UV-A crosslinking on porcine corneal mechanical anisotropy with optical coherence elastography," Biomedical Optics Express, vol. 8, no. 1, pp. 349-366, 2017.

[11] M. Singh, J. Li, Z. Han et al., "Investigating elastic anisotropy of the porcine cornea as a function of intraocular pressure with optical coherence elastography," Journal of Refractive Surgery, vol. 32, no. 8, pp. 562-567, 2016.

[12] A. Elsheikh and D. Alhasso, "Mechanical anisotropy of porcine cornea and correlation with stromal microstructure," Experimental Eye Research, vol. 88, no. 6, pp. 1084-1091, 2009.

[13] S. S. Khachikian, M. W. Belin, and J. B. Ciolino, "Intrasubject corneal thickness asymmetry," Journal of Refractive Surgery, vol. 24, no. 6, pp. 606-609, 2008.

[14] C. Boote, S. Hayes, M. Abahussin, and K. M. Meek, "Mapping collagen organization in the human cornea: left and right eyes are structurally distinct," Investigative Opthalmology \& Visual Science, vol. 47, no. 3, pp. 901-908, 2006.

[15] W. Sekundo, K. S. Kunert, and M. Blum, "Small incision corneal refractive surgery using the small incision lenticule extraction (SMILE) procedure for the correction of myopia and myopic astigmatism: results of a 6 month prospective study," British Journal of Ophthalmology, vol. 95, no. 3, pp. 335-339, 2011.

[16] C. Xue, Y. Xiang, M. Shen et al., "Preliminary investigation of the mechanical anisotropy of the normal human corneal stroma," Journal of Ophthalmology, vol. 2018, Article ID 5392041, 7 pages, 2018.

[17] L. A. Davis, S. E. Stewart, C. G. Carsten, B. A. Snyder, M. A. Sutton, and S. M. Lessner, "Characterization of fracture behavior of human atherosclerotic fibrous caps using a miniature single edge notched tensile test," Acta Biomaterialia, vol. 43, pp. 101-111, 2016.

[18] Y. Zeng, J. Yang, K. Huang, Z. Lee, and X. Lee, “A comparison of biomechanical properties between human and porcine cornea," Journal of Biomechanics, vol. 34, no. 4, pp. 533-537, 2001.

[19] A. Vestergaard, A. R. Ivarsen, S. Asp, and J. Ø. Hjortdal, "Small-incision lenticule extraction for moderate to high myopia: predictability, safety, and patient satisfaction," Journal of Cataract \& Refractive Surgery, vol. 38, no. 11, pp. 2003-2010, 2012.

[20] C. Albou-Ganem, A. Lavaud, and R. Amar, "Smile :correction de la myopie par ablation réfractive par laser femtoseconde 
d'un lenticule intracornéen," Journal Français d'Ophtalmologie, vol. 38, no. 3, pp. 229-237, 2015.

[21] D. Z. Reinstein, G. I. Carp, T. J. Archer, and M. Gobbe, "Outcomes of small incision lenticule extraction (SMILE) in low myopia," Journal of Refractive Surgery, vol. 30, no. 12, pp. 812-818, 2014.

[22] S. Arba Mosquera and S. Verma, "Bilateral symmetry in vision and influence of ocular surgical procedures on binocular vision: a topical review," Journal of Optometry, vol. 9, no. 4, pp. 219-230, 2016.

[23] F. Cavas-Martínez, D. Piñero, D. Fernández-Pacheco, J. Mira, F. Cañavate, and J. Alió, "Assessment of pattern and shape symmetry of bilateral normal corneas by scheimpflug technology," Symmetry, vol. 10, no. 10, p. 453, 2018.

[24] X. Zheng, F. Bao, B. Geraghty et al., "High intercorneal symmetry in corneal biomechanical metrics," Eye Vis (Lond), vol. 3, p. 7, 2016.

[25] D. Amato, F. Oddone, M. Nubile, R. A. Maria Colabelli Gisoldi, C. M. Villani, and A. Pocobelli, "Pre-cut donor tissue for Descemet stripping automated keratoplasty: anterior hinged lamella on versus off," British Journal of Ophthalmology, vol. 94, no. 4, pp. 519-522, 2010.

[26] G. Wollensak, E. Spoerl, and T. Seiler, "Stress-strain measurements of human and porcine corneas after riboflavinultraviolet-A-induced cross-linking," Journal of Cataract \& Refractive Surgery, vol. 29, no. 9, pp. 1780-1785, 2003.

[27] A. Elsheikh, D. Wang, M. Brown, P. Rama, M. Campanelli, and D. Pye, "Assessment of corneal biomechanical properties and their variation with age," Current Eye Research, vol. 32, no. 1, pp. 11-19, 2007.

[28] T. T. Andreassen, A. Hjorth Simonsen, and H. Oxlund, "Biomechanical properties of keratoconus and normal corneas," Experimental Eye Research, vol. 31, no. 4, pp. 435-441, 1980.

[29] D. H. Glass, C. J. Roberts, A. S. Litsky, and P. A. Weber, “A viscoelastic biomechanical model of the cornea describing the effect of viscosity and elasticity on hysteresis," Investigative Opthalmology \& Visual Science, vol. 49, no. 9, pp. 3919-3926, 2008.

[30] T. Seiler, M. Matallana, S. Sendler, and T. Bende, "Does Bowman's layer determine the biomechanical properties of the cornea?" Refract Corneal Surgery, vol. 8, no. 2, pp. 139$142,1992$.

[31] A. Elsheikh, D. Alhasso, and P. Rama, "Assessment of the epithelium's contribution to corneal biomechanics," Experimental Eye Research, vol. 86, no. 2, pp. 445-451, 2008.

[32] C. Labate, M. Lombardo, M. P. De Santo, J. Dias, N. M. Ziebarth, and G. Lombardo, "Multiscale investigation of the depth-dependent mechanical anisotropy of the human corneal stroma," Investigative Opthalmology \& Visual Science, vol. 56, no. 6, pp. 4053-4060, 2015.

[33] J. Matalia, M. Francis, P. Gogri, P Panmand, H Matalia, and A Sinha Roy, "Correlation of corneal biomechanical stiffness with refractive error and ocular biometry in a pediatric population," Cornea, vol. 36, no. 10, pp. 1221-1226, 2017.

[34] Y. Hon, G.-Z. Chen, S.-H. Lu, D. C. C. Lam, and A. K. C. Lam, "High myopes have lower normalised corneal tangent moduli (less 'stiff corneas) than low myopes," Ophthalmic and Physiological Optics, vol. 37, no. 1, pp. 42-50, 2017.
[35] A. Plakitsi, C. O’Donnell, M. A Miranda, W. N. Charman, and H. Radhakrishnan, "Corneal biomechanical properties measured with the Ocular Response Analyser in a myopic population," Ophthalmic and Physiological Optics, vol. 31, no. 4, pp. 404-412, 2011.

[36] M. Shen, F. Fan, A. Xue, J. Wang, X. Zhou, and F. Lu, "Biomechanical properties of the cornea in high myopia," Vision Research, vol. 48, no. 21, pp. 2167-2171, 2008. 